\title{
Application driven, AMC-based cross-layer optimization for video service over LTE
}

\author{
Yongil Kwon*, Doug Young Suh, Sung Chun Kim and Een Kee Hong
}

\begin{abstract}
In this paper, we propose a cross-layer optimization scheme in which the application layer controls the medium access network (MAC) and physical (PHY) layers in long-term evolution (LTE, from 3rd generation partnership project [3GPP] release 8) to maximize the quality of video streaming services. We demonstrate how to optimize quality using the equi-signal-to-noise ratio (equi-SNR) from the lower layer and the equi-peak signal-to-noise ratio (equi-PSNR) from the upper layer in the two-dimensional domain, consisting of a bit rate $(R)$ and packet loss ratio (PLR). The proposed approach outperforms the conventional approach, which operates regardless of the application-specific requirements for quality of service (QoS) and quality of experience (QoE) in PHY.
\end{abstract}

Keywords: SVC, AMC, CLO, QOS, LTE

\section{Introduction}

User demand for mobile multimedia services has exploded. However, current mobile multimedia services have weaknesses such as fading, congestion, insufficient resources, and time-varying conditions. These problems need to be addressed. Studies on improving (QoS) can be classified into three categories: [1]. real-time video service optimization based on wireless channel states; [2]. wireless resource allocation based on video characteristics; and [3] a hybrid of categories [1] and [2].

The authors of references [1-3] proposed scheduling and allocation methods using the available mechanisms and parameters in the medium access network (MAC)/ physical (PHY) layers of wireless networks. In addition, Fang [4] and $\mathrm{Ha}[5]$ improved the service quality by considering packet loss using cross-layer optimization (CLO) between whole layers.

Video is made up of packets with different priorities. Average video quality could be adaptively improved by protecting the more important packets from error and filtering out less important packets at a low bit rate $(R)$. The cross-layer methods mentioned above adapt the video layer to already-determined MAC/PHY conditions.

Even under the same mobile conditions, however, various combinations of ( $R$, packet loss ratio [PLR]) are possible based on the choice of modulation and

* Correspondence: pigsoon012@gmail.com Kyung-Hee University, Suwon, Korea

(c) 2011 Kwon et al; licensee Springer. This is an Open Access article distributed under the terms of the Creative Commons Attribution License (http://creativecommons.org/licenses/by/2.0), which permits unrestricted use, distribution, and reproduction in any medium, provided the original work is properly cited. channel-coding scheme. If the target block error rate (BLER) is set too low, the available bit rate will also be low. Since most mobile channels have fixed transmission parameters suitable for non-real-time data services, it is important that MAC/PHY parameters are chosen differently, based on the service requirements of real-time video services.

Haghani [6] suggested a method of improving video quality by classifying the significance of frames in a video stream and transmitting them as packets of different priorities that correspond to those in IEEE 802.16 QoS classes. In referenced paper [7], a method that allocates bit rate by predicting the quality of the video after recovery from packet losses along the wireless channel was suggested. This method searches for the optimal point yielding the best video quality using various rate control methods, such as fine granular scalability (FGS) or H.264/MPEG-4 scalable video coding (SVC). FGS guarantees apropos degradation, but its rate-distortion $(R-D)$ performance is so poor that it has become obsolete.

We focused on a third method for improving QoS. At a signal-to-noise ratio (SNR) measured in the lower layers, all possible combinations of ( $R$, PLR) for all possible modulation and coding scheme (MCS) levels yield the equi-SNR graph. The upper layer (including the video layer and transport layer) provides equi-PSNR graphs, which are also sets of ( $R$, PLR) combinations, 
and result in the same PSNR. An optimal point can be found at the highest PSNR that lies on the equi-SNR graph of the current SNR. The optimal operation point is determined to be its nearest MCS point along the selected equi-SNR graph. This enables the highest PSNR achievable for a given set of mobile channel conditions. Since both equi-SNR and equi-PSNR graphs are independently prepared, the computational burden can be dramatically reduced.

Section 2 describes related background technologies in LTE and SVC. Section 3 introduces the proposed application-driven adaptive modulation and coding (AMC) scheme. The performance of the proposed method is demonstrated using experiments in Section 4. Section 5 concludes this paper.

\section{Background}

Following CLO, in this paper, the lower layers are based on 3GPP LTE [8], which includes AMC and hybrid-auto repeat request $(\mathrm{H}-\mathrm{ARQ})$, while the upper layers use forward error correction (FEC) and H.264/MPEG-4 SVC video streams.

\subsection{MAC and PHY layers in 3GPP LTE}

Available bit rate $(R)$ and BLER $p_{b}$ are determined according to the SNR between the node base transceiver station (Node B) and the user equipment (UE). Bit rate in the PHY layer is determined by an MCS including $\mathrm{H}-$ ARQ.

The symbol rates of quadrature phase shift keying (QPSK), 16 quadrature amplitude modulation (QAM), and 64QAM are 2:4:6, and their symbol block sizes are 480,960 , and 1440 bits, respectively. Coding rates range from 0.3 to 0.8 . A combination of a modulation mode and a coding rate is called a MCS. The MCS level is selected adaptively according to a predefined target BLER and time-varying channel quality information (CQI), particularly SNR [8]. As we can see in Table 1 there are five CQI levels. Block size $m_{c}$ is determined by CQI level $c$.

\section{Table 1 CQI table and BLER correspond to SNR}

\begin{tabular}{llllll}
\hline CQI index $(\boldsymbol{c})$ & $\mathbf{5}$ & $\mathbf{1 0}$ & $\mathbf{1 5}$ & $\mathbf{2 0}$ & $\mathbf{2 5}$ \\
\hline Modulation & QPSK & 16QAM & 16QAM & 64QAM & 64QAM \\
\hline Coding rate & 0.36 & 0.33 & 0.6 & 0.55 & 0.8 \\
\hline $\begin{array}{l}\text { Block size } \\
\text { (bits/block) }\end{array}$ & 152 & 296 & 552 & 840 & 1192 \\
\hline SNR (S) & \multicolumn{5}{c}{ BLER (after H-ARQ } \\
\hline 5 & 0.013 & 0.062 & 0.231 & 0.358 & 0.591 \\
\hline 10 & 0.002 & 0.013 & 0.031 & 0.089 & 0.177 \\
\hline 15 & 0.0002 & 0.0028 & 0.006 & 0.018 & 0.037 \\
\hline 20 & 0.0002 & 0.001 & 0.001 & 0.005 & 0.009 \\
\hline
\end{tabular}

A block is the minimum transmission unit of orthogonal frequency division multiplexing (OFDM), while a packet is the minimum transmission unit in the transport layer. If the size of a packet is larger than that of a block, the packet may be segmented into blocks in the transmitter and assembled in the receiver. If the size of a packet is $\mathrm{M}$, it is divided into $n=\left[\left(M+m_{c}-1\right) / m_{c}\right]$ blocks. As suggested in [1], a damaged block and its corresponding packet are assumed to be discarded, so that the PLR of the PHY layer is

$$
P_{\mathrm{PHY}, c}(S, N)=1-\left(1-P_{\mathrm{b}}(S, c)\right)^{n},
$$

where $P_{\mathrm{b}}(S, c)$ is BLER and $n$ is the number of blocks in a packet. For a given SNR $(S)$ and video packet size, a set of $\left(R, P_{\mathrm{PHY}}\right)$ is determined by AMC (Figure 1$)$.

\subsection{SVC and FEC}

Rate control and unequal error protection techniques are used for adaptation to $\left(R, P_{\mathrm{PHY}}\right)$ provided by the MAC/PHY layers. SVC [9] is useful because it can be used for simultaneously encoding video streams and includes more kinds of scalability, such as spatial scalability and quality scalability.

Figure 2 shows a case in which there are six layers with a combination of two spatial scalability layers and three temporal scalability layers. (Quality $[Q]$ scalability is not used.) The spatial and temporal resolutions of the base layer (the lowest layer) are quarter common-intermediate-format (QCIF, $176 \times 144$ ) and $15 \mathrm{~Hz}$, while those of the highest layer are CIF $(352 \times 288)$ and 30 $\mathrm{Hz}$. The priority of the lower layer is higher than that of the upper layer, since the upper layer will not be decoded correctly, if the lower layer is lost.

Figure 2 shows rate-distortion curves for video sequences encoded in the SVC architecture. The dynamic range in bit rate ranges from $134 \mathrm{kbps}$ to more than $600 \mathrm{kbps}$. The PSNR of a missing picture is calculated by comparing the original picture with the temporally nearest decoded picture. Since PSNR is calculated in CIF size, the base layer image has to be up-sampled before PSNR calculation.

$R S\left(N, K, P_{\mathrm{PHY}}\right)$ is the residual PLR in the application layer. $P_{\text {app }}$ is calculated depending on the coding ratio $K / \mathrm{N}$ and number of video packets $K$. Through the $R S(N$, $K$, $\mathrm{PHY}), P_{\mathrm{PHY}}$ becomes $P_{\text {app }}$ as follows:

$$
R S\left(N, K, P_{\mathrm{PHY}}\right)=P_{\mathrm{app}}=\left(\sum_{j-N-K+1}^{N}\left(\begin{array}{c}
N \\
j
\end{array}\right) P_{\mathrm{PHY}}^{j}\left(1-P_{\mathrm{PHY}}\right)^{N-j}\right) / N,
$$

where $N$ is the total number of transported packets, including both video and parity packets. $K$ and $N$ are selected to maximize $K$ by satisfying the constraints $P_{\text {app }}$ ${ }_{<} P_{\text {target }}$ and $R \times \frac{T}{S}>M N($ bytes $)$, where $T$ is a group 


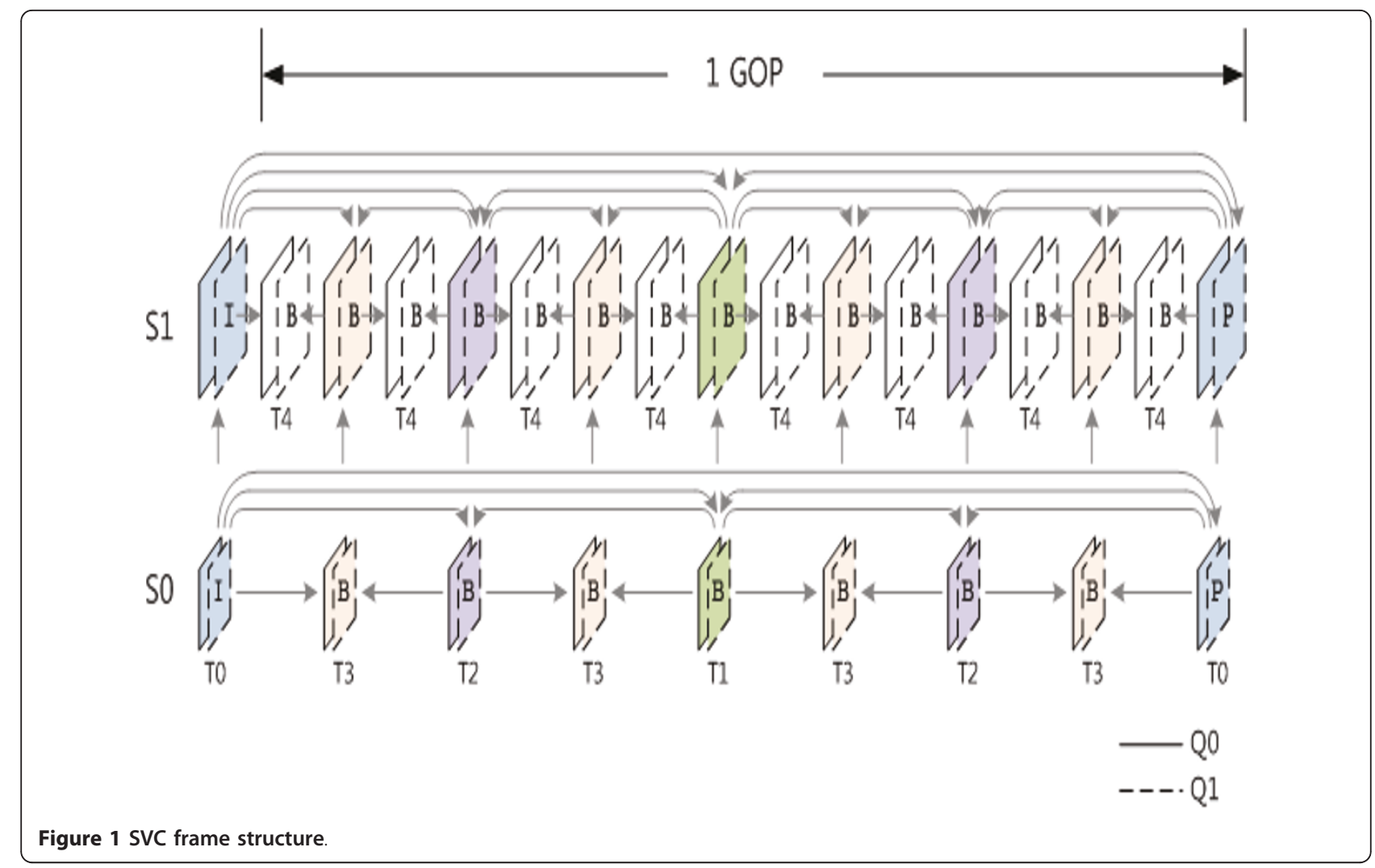

of pictures (GOP) of the period in seconds and $M$ is the packet size in bytes. In this paper, $P_{\text {targe }}=10^{-5}$. (If $T=1$ $\mathrm{S}$ and $P_{\text {targe }}=10^{-5}$, and the mean time between failures is $10^{5} \mathrm{~s}$, an outage is expected once every day on average.)

\section{Application-driven AMC}

We propose a cross-layer optimization method where $\mathrm{AMC}$ is driven by the application layer, i.e., the video

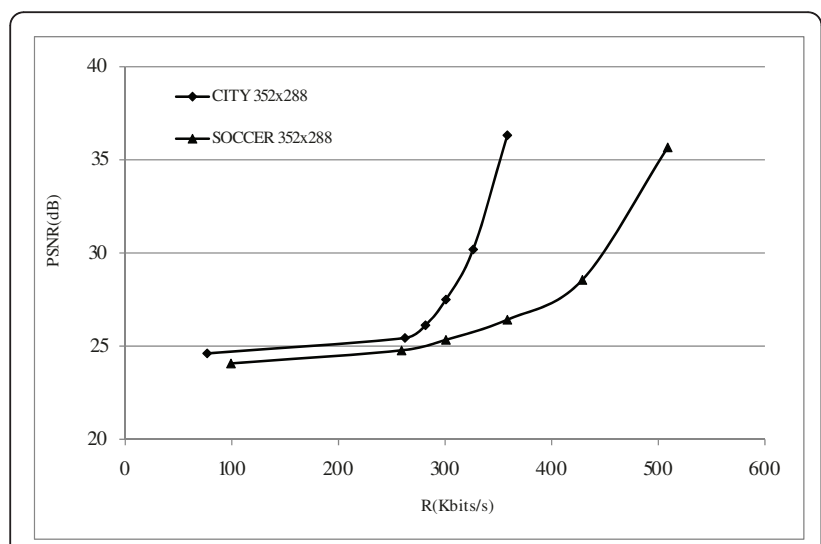

Figure 2 R-D curve (frame rate: enhance $30 \mathrm{~Hz}$, base $15 \mathrm{~Hz}$, QP: enhance 28, base 30 , resolution: enhance CIF, base QCIF, contents: Soccer, City sequence). service. In the same two-dimensional space of ( $R, \mathrm{PLR})$, equi-SNR curves are generated by the PHY layer, while equi-PSNR curves are generated by the application layer. Using two sets of curves, the MCS which enables the highest PSNR can be selected for a given SNR.

\subsection{Generation of the equi-SNR curve}

Figure 3 shows that $P_{\mathrm{PHY}}$ is determined by SNR from the MAC/PHY layer and packet size $M$, where $M$ denotes the packet size of video data. We assume that the bit rate of the video stream is constant. Using the results of PLR and the CQI table, we can define a set of bit rate $R$ and PLR $P_{\mathrm{PHY}}\left(R, P_{\mathrm{PHY}}\right)$, as a vector $\bar{v}$ in the two-dimensional $R$ $P_{\text {PHY }}$ space. Conventionally, only one v is selected as an operation point with respect to the predefined target BLER. For a given SNR and a given maximum retransmission number of $\mathrm{H}$-ARQ, however, at most five different $\overline{\mathrm{V}}$ 's can be used, since $|C|=5$, and the set of $\bar{v}$ 's is defined as the equi-SNR curve. For immediate adaptation in a time-varying condition, there are sufficient SNR values; these equi-SNR curves can be generated before providing video service in the $R-P_{\mathrm{PHY}}$ space.

Figure 4 gives an equi-SNR graph of all possible operation points at every given SNR. Among them, only one point, bigger than the others, is selected by the conventional scheme. It is questionable whether the selection is good for any application. 


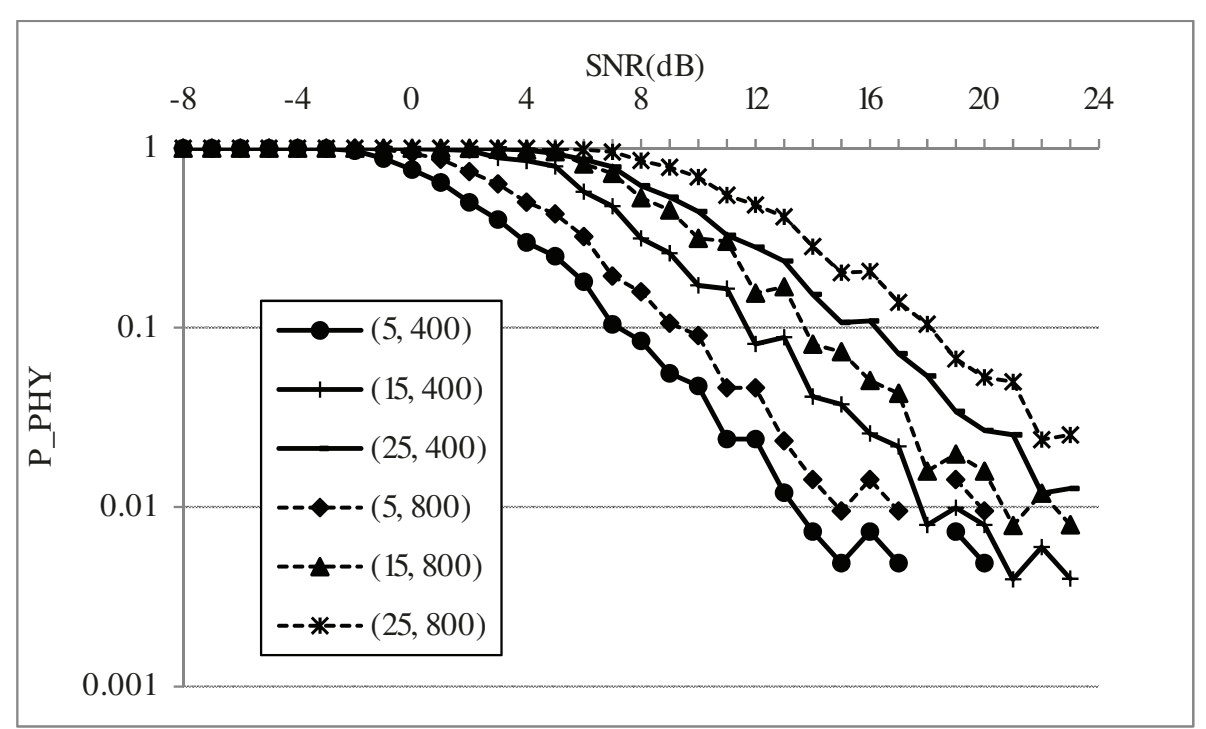

Figure 3 PLR of each packet size.

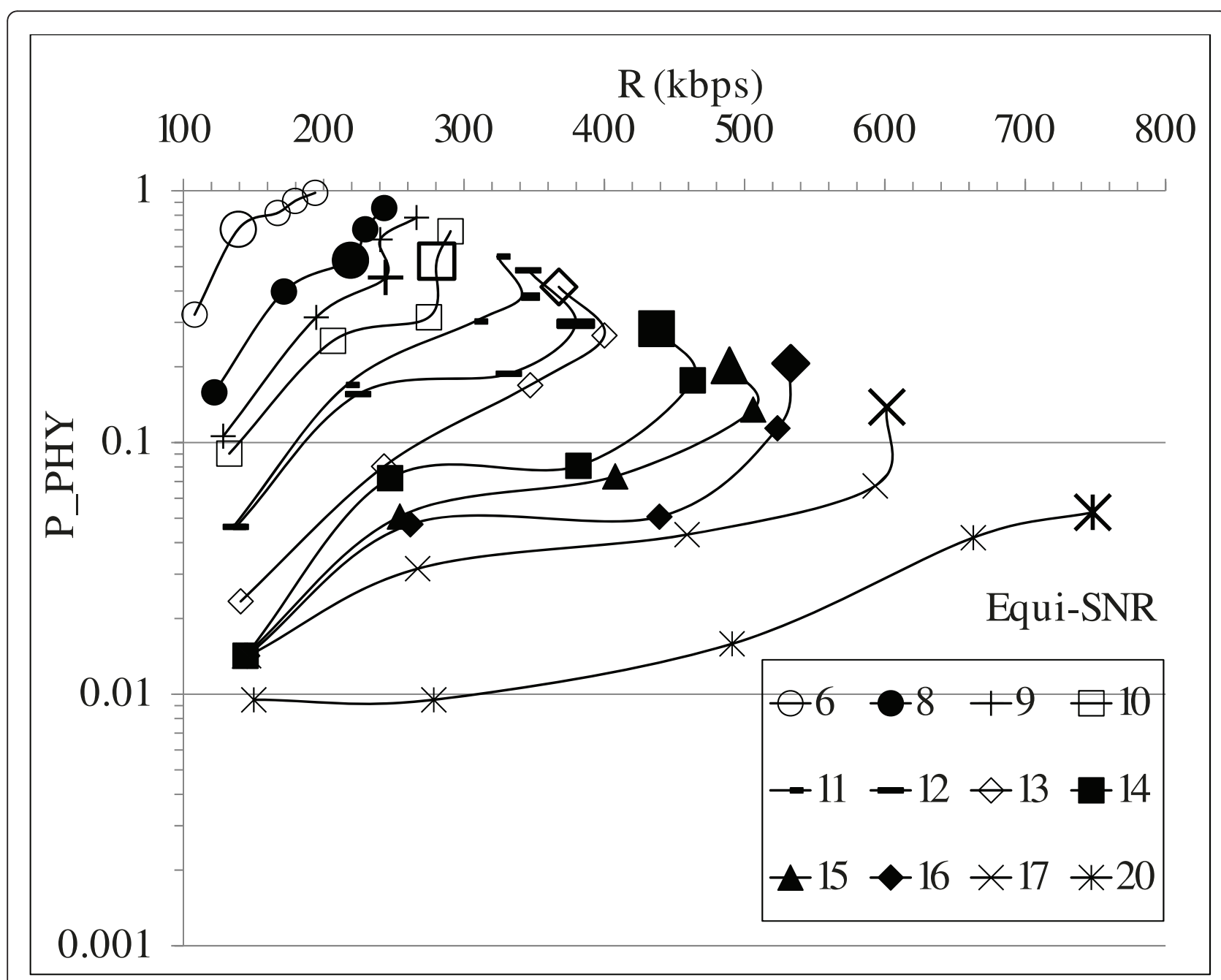

Figure 4 Equi-SNR graph (CQI(c): 5-25, SNR: 6-20) 


\subsection{Generation of the equi-PSNR curve}

In the same $R-P_{\text {PHY }}$ space, video service range (VSR) $\check{R}$ can be represented two-dimensionally, as shown in Figure 5. $\check{R}$ includes the bit rates of both video packets and parity packets. If $P_{\mathrm{PHY}}$ is almost zero, no parity is included and all bit rates are used for video data. $\check{R}$ corresponds to the top line from $r_{\min }$ to $r_{\max }$. As $P_{\mathrm{PHY}}$ increases (vertically moving down), parity data are added to satisfy the loss by constraint $P_{\text {app }}<\mathrm{P}_{\text {target }}$. This results in exponentially decreasing lines on both sides.

$$
\check{R}=\left\{\left(R, P_{\text {PHY }}\right) \mid r>\frac{r N}{K}, R S\left(N, K, P_{\text {PHY }}\right)=P_{\text {app }} \leq P_{\text {targer }} \quad K=\frac{\tau \times T}{M \times 8(\text { bits per byles })}, \quad r_{\min } \leq r \leq r_{\max }\right\}
$$

Within the $\check{R}$, we can calculate the maximum PSNR at each $R, P_{\mathrm{PHY}}$ point. Then, the set of points representing the same PSNR is defined as the equi-PSNR curve. Figure 5 shows equi-PSNR curves of the lowest and highest PSNR values, while Figure 6 shows all equi-PSNR graphs as a contour map.

\subsection{Application-driven AMC}

The equi-SNR curve for a given SNR value is the trace of all possible sets of $\left(R, P_{\mathrm{PHY}}\right)$, while the equi-PSNR curve for a given PSNR value is a trace of $\left(R, P_{\mathrm{PHY}}\right)$ sets that result in the video quality of the given PSNR when FEC is optimally applied. Both equi-SNR curves and equi-PSNR curves are drawn in the two-dimensional $(R$, $\left.P_{\mathrm{PHY}}\right)$ space. As we mentioned in the introduction, an optimal operation point is found by overlapping those two sets of curves. For each measured SNR value, an optimal service point $\left(R, P_{\mathrm{PHY}}\right)$ can be found if at least one equi-SNR curve exists in the VSR and the equi-SNR and equi-PSNR curves are convex. An equi-SNR curve $\bar{V}$ has connections for discrete points $\bar{v}$ for all CQI levels.

$$
\bar{V}(S, M, A)=\left(\bar{v} \mid \bar{v}_{c}(S, M, A)=\left\{R_{c} P_{\mathrm{PHY}, c}\right\}, \quad c=5,10, \ldots, 25\right),
$$

where c is a CQI level, $M$ is packet size, $S$ is SNR value, and $A$ is the number of allocated resource blocks ( $A=1$ in this paper).

An equi-PSNR curve with a PSNR of $\mathrm{q}$ is defined as

$$
\bar{Q}(q)=\left\{\left(R, P_{\mathrm{PHY}}\right) \mid q=\max _{(\mathrm{sl}, \mathrm{fl})} \operatorname{PSNR}\left(R, P_{\mathrm{PHY}}\right)\right\},
$$

where sl and fl represent scalability level and parity level (i.e., FEC level), respectively. The bit rates of video data are determined by sl. Sums of bit rates of video data and parity data should not exceed $R$, and residual PLR resulting from $\mathrm{fl}$ should be less than the target PLR $P_{\text {target }}$ when PLR resulting from the MAC/PHY layers is $P_{\mathrm{PHY}}$. Using these two kinds of curves in the $\left(R, P_{\mathrm{PHY}}\right)$ space, the optimal operation point can be identified.

$$
\begin{aligned}
& \left(R_{c^{*}, P_{\mathrm{PHY}}^{*}}\right)=\arg \max q\left(\bar{v}_{s}\right) \\
& \bar{v}=\bar{V}(S, M, A)
\end{aligned}
$$

At the same time, the optimal CQI level c* can be determined. This operation point provides the best video quality under certain conditions, which are SNR, packet size, and number of resource blocks.

\section{Experiments and discussion}

The video sequences "City" and "Soccer" were used for experiments. Since spatial complexity of both sequences is high while the temporal complexity of "Soccer" is much higher than that of "City", the highest quality of

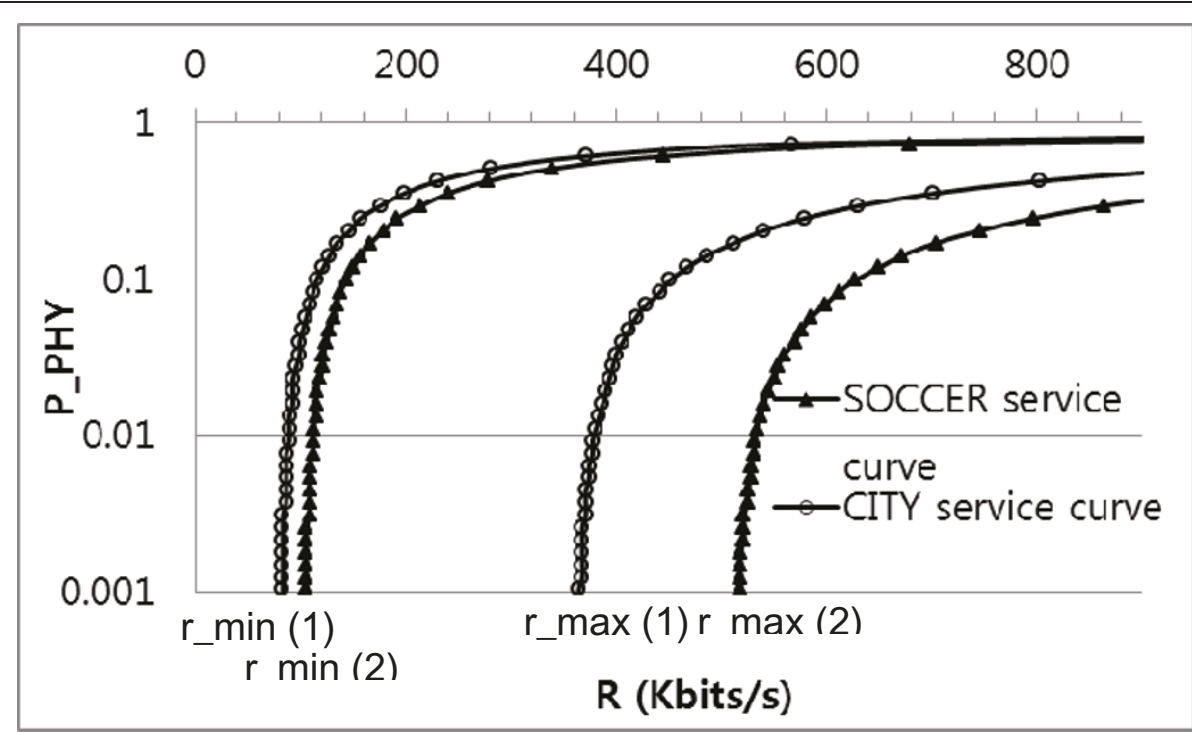

Figure 5 Video service range: minimum video stream rate line $\left(r_{-}\right.$min) and maximum video rate line $\left(r_{-} \max \right)$ of (1) City, (2) Soccer. 


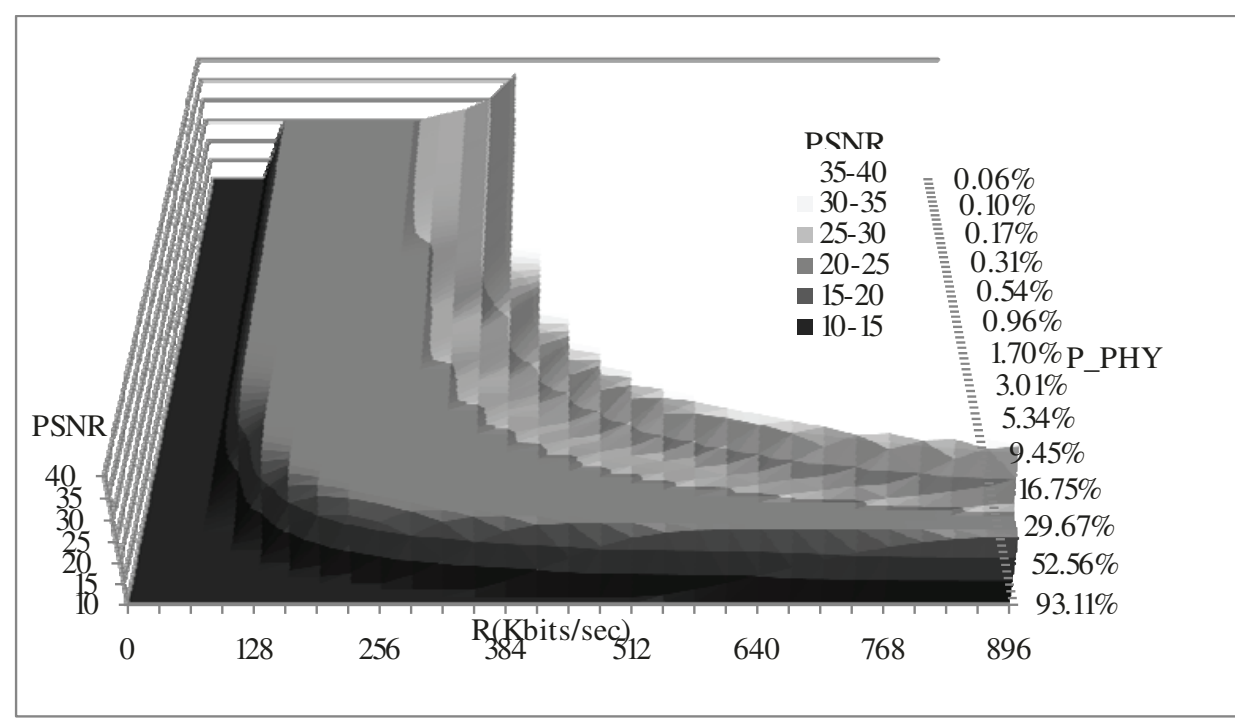

(a) Soccer sequence

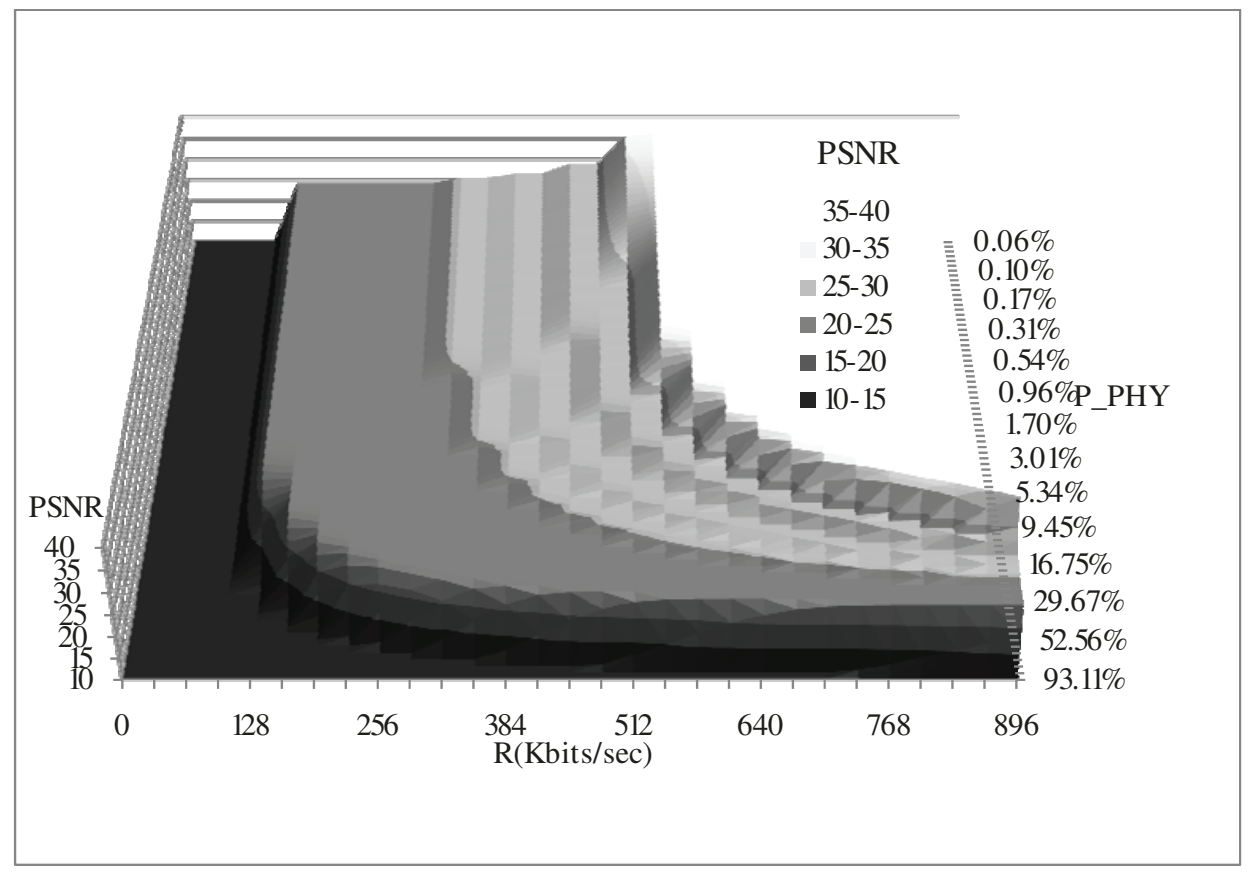

Figure 6 PSNR over $\left(R, P_{\mathrm{PHY}}\right)$ from the application layer. (a) Soccer sequence. (b) City sequence.

"City" is achieved at lower bitrate as shown Figure 2. Therefore, their video service areas vary, as shown in Figure 5 in Section 3.2. They are encoded in six layers, including two spatial layers and five temporal layers (the base has four temporal layers). The two spatial layers are composed of QCIF and CIF, while the three temporal layers have frame rates from 15 to $30 \mathrm{~Hz}$. Video packet size is fixed at $M=400$ bytes and $P_{\text {target }}$, which is the target PLR in the application layer, is $10^{-5}$.
Figure 6 shows contour maps of equi-PSNR curves for City and Soccer, respectively. The highest plateaus at the upper and right corners correspond to video quality when all layers are correctly received and decoded. Gray regions represent VSR. As the video service area of each sequence is different, the slope of their contour line is also different. These differences show distinct characteristics when equi-SNR and equiPSNR curve are merged. 
Figures 4 and 6 are merged into Figure 7. The equiSNR curves in Figure 4 are drawn in white-colored lines. The equi-PSNR curves are denoted as a blackand-white contour map in which a brighter region means higher video quality. In this graph, the white points are conventional points according to the target BLER $\left(10^{-1}\right)$. We can find four other points that have different PSNRs. In the VSR, the vector of maximum VSR points is defined as $\overline{\mathrm{MCS}}=\bar{r}\left(r=r_{\max }\right) \in R$. The operation points nearest to $\overline{\mathrm{MCS}}$ yield the best PSNR quality. We derive the optimal CQI point $\mathrm{c}^{*}$ as follows:

$$
c^{*}=\min \left|\overline{\mathrm{MCS}}-\bar{v}_{\mathrm{s}}\right| \text {. }
$$

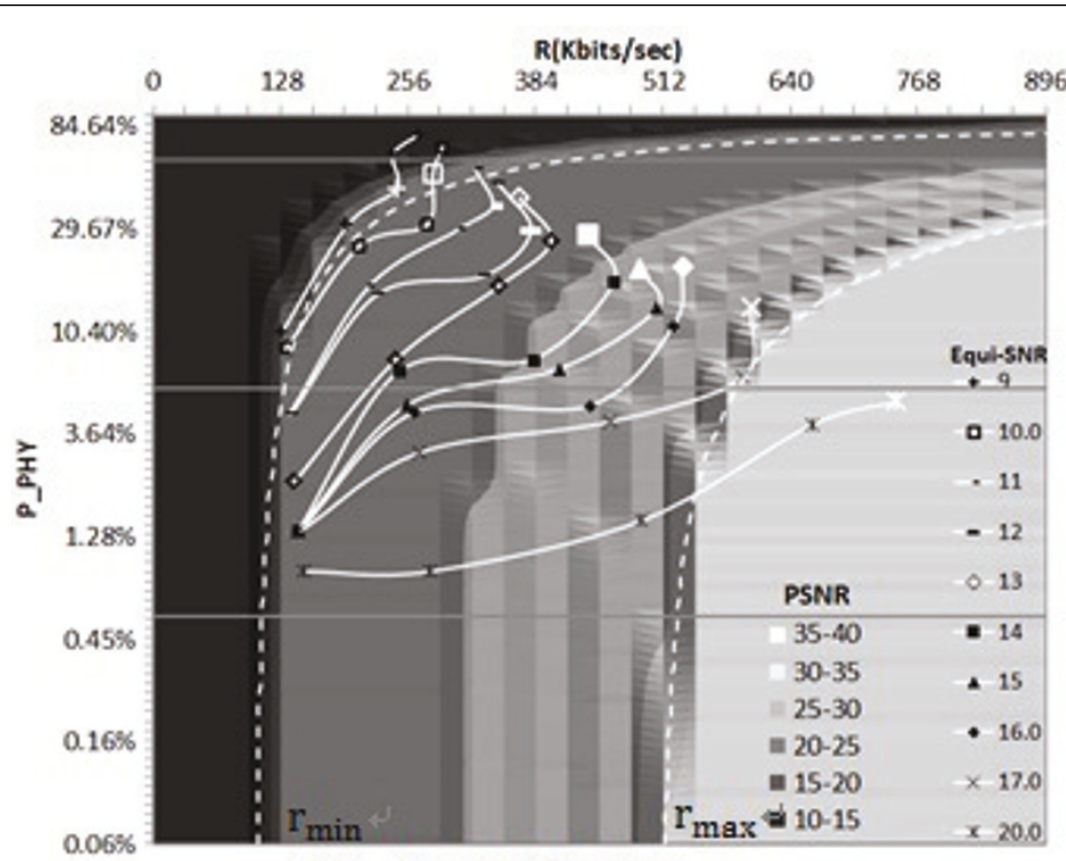

(a) Soccer sequence

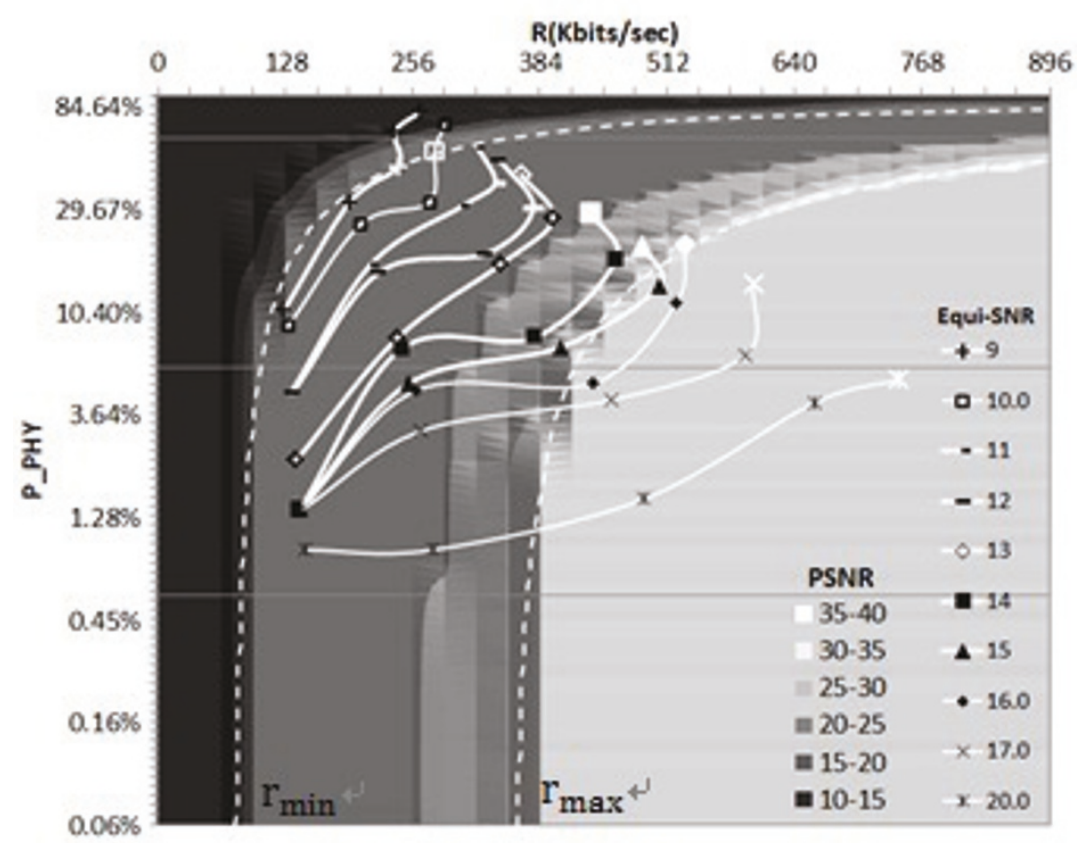

(b) City sequence

Figure 7 PSNR and equi-SNR, conventional point, VSR. (a) Soccer sequence. (b) City sequence. 
$\mathrm{AD}-\mathrm{AMC}$ ensures maximum video quality in timevarying situations. The conventional MCS selection scheme is not optimal for a video service, which is less sensitive to information loss and requires a higher bit rate than a normal data service with the same conditions. In this paper, we used only one resource block (i.e., $A=1$ ). For video of higher resolution, more than one resource block $(A>1)$ providing a higher bit rate will be used. The optimal CQI point at the maximum bit rate $(R)$ must be selected for the Soccer sequence, as it has a wider video service range than the City sequence does; furthermore, a resource-saving selection must be made for the City sequence, as it is in an area where the equi-SNR curve is over the limit.

As shown in Figure 8, at the two extremes of the highest SNR and lowest SNR, the proposed method does not seem to have any gain while the UE, the mobile device in LTE, moves in the cell area. At the lowest SNR, even the lowest-quality video cannot be delivered at all. At the highest SNR, resources are so abundant that all video data can be delivered. In a normal situation with an SNR range from 12 to $20 \mathrm{~dB}$, the proposed method outperforms the conventional MCS selection scheme by 2 to $3 \mathrm{~dB}$ in terms of PSNR.

\section{Conclusions}

This paper proposes an active cross-layer design in which the application layer controls MAC/PHY operation. MAC/PHY operation is currently controlled to maximize the channel utility of non-real-time data services. For higher total throughput, channel resources may be consumed primarily by a few terminals whose SNRs are high enough, while others "starve." The service requirements of real-time multimedia services, however, are different from those of non-real-time services. The real-time service should regard characteristics of the video sequence over ( $R$, PLR) rather than use a fixed target BLER, since each sequence has the same condition set. As mobile multimedia services become more popular, operation policies must adapt to their demands.

We have demonstrated the effects of application-driven MAC/PHY operation, in which modulation type and channel-coding level are determined to maximize QoS. Among the possible operation points at a certain SNR, maximizing PSNR is selected as an operation point that satisfies the BLER constraint regardless of application. In most cases, operation points for multimedia services are selected at a higher bit rate and higher BLER compared to those of non-real-time services. By virtue of scalable video coding and FEC for the recovery of lost packets, the proposed method achieved at most a $5 \mathrm{~dB}$ gain in PSNR.

We also described a technique to isolate the lower layers from the upper layers of the system without losing the benefits of cross-layer optimization by simplifying the interfaces between the two. Both equi-PSNR curves from the application and transport layers and equi-SNR curves from the MAC/PHY layers are mapped

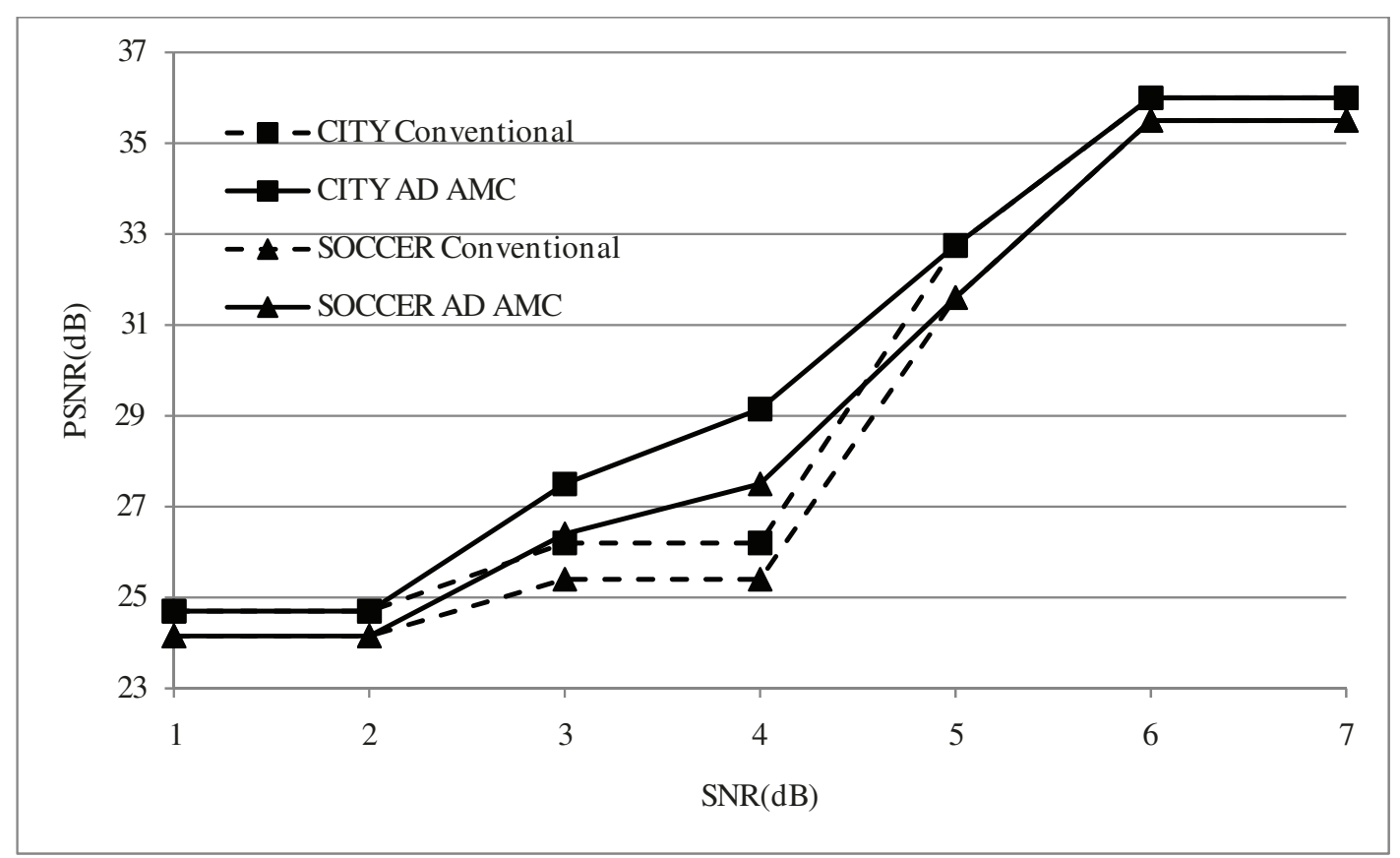

Figure 8 Average PSNR of UE moving in the cell area. 
onto the same two-dimensional space of $\left(R, P_{\mathrm{PHY}}\right)$. The equi-PSNR curves in the $\left(R, P_{\mathrm{PHY}}\right)$ space can be prepared independently of the equi-SNR curves, and vice versa. By using these two kinds of curves, which are prepared before service, cross-layer optimization during service is simply used to measure SNR and to determine a maximum PSNR point along the corresponding equiSNR curve. Even though the MAC/PHY scheme has been altered so that new equi-SNR curves are built, the same equi-PSNR curves can be used with the new equiSNR curves. This approach will enable users to switch video-coding techniques or to switch mobile communication modality more easily in the further development of cross-layer design.

\section{Abbreviations}

AMC: adaptive modulation and coding; BLER: block error rate; CLO: crosslayer optimization; CQl: channel quality information; FEC: forward error correction; FGS: fine granular scalability; GOP: group of pictures; $\mathrm{H}-\mathrm{ARQ}$ : hybrid-auto repeat request; MAC: medium access network; MCS: modulation and coding scheme; OFDM: orthogonal frequency division multiplexing; PLR: packet loss ratio; QAM: quadrature amplitude modulation; QCIF: quarter common-intermediate-format; QoE: quality of experience; QoS: quality of service; QPSK: quadrature phase shift keying; SNR: signal-to-noise ratio; SVC: scalable video coding; VSR: video service range.

\section{Acknowledgements}

This paper was partly supported by the IT R\&D program of MKE/KEIT (KI001814, Game Theoretic Approach for Crosslayer Design in Wireless Communications) and MKE (The Ministry of Knowledge Economy), Korea, under the ITRC (Information Technology Research Center) support program supervised by the NIPA (National IT Industry Promotion Agency) (NIPA-2011(C1090-1111-0001)).

\section{Competing interests}

The authors declare that they have no competing interests.

Received: 6 September 2010 Accepted: 7 July 2011

Published: 7 July 2011

\section{References}

1. S Shankar, M van der Schaar, Performance analysis of video transmission over IEEE 802.11a/e WLANs. IEEE Trans Veh Technol. 56(4), 2346-2362 (2007)

2. F Foukalas, $V$ Gazis, Cross-layer design proposals for wireless mobile networks: a survey and taxonomy. IEEE Commun Surv. 10(1), 70-85 (2008)

3. M Van der Schaar, Cross-layer wireless multimedia transmission: challenges, principles, and new paradigms. IEEE Wirel Commun Mag. 12(4), 55-58 (2005)

4. T Fang, L Chau, GOP-based channel rate allocation using genetic algorithm for scalable video streaming over error-prone networks. IEEE Trans Image Process. 15(6), 1323-1330 (2006)

5. $\mathrm{H} \mathrm{Ha}, \mathrm{C}$ Yim, Layer-weighted unequal error protection for scalable video coding extension of H.264/AVC. IEEE Trans Consumer Electron. 54(2), 736-744 (2008)

6. E Haghani, S Parekh, A quality-driven cross-layer solution for MPEG video streaming over WiMAX networks. IEEE Trans Multimedia. 11(6), 1140-1147 (2009)

7. MK Jubran, M Bansal, Accurate distortion estimation and optimal bandwidth allocation for scalable H.264 video transmission over MIMO systems. IEEE Trans Image Process. 18(1), 106-116 (2009)

8. S Sesia, I Toufik, LTE, the UMTS long term evolution: from theory to practice. (2009) ISBN 978-0-470-69716-0

9. Text of ISO/IEC 14496-4:2001/PDAM 19 Reference Software for SVC, Joint Video Team (JVT) of ISO-IEC MPEG \& ITU-T VCEG, N9195 (2007) doi:10.1186/1687-1499-2011-31

Cite this article as: Kwon et al:: Application driven, AMC-based crosslayer optimization for video service over LTE. EURASIP Journal on Wireless Communications and Networking 2011 2011:31.

\section{Submit your manuscript to a SpringerOpen ${ }^{\mathcal{O}}$ journal and benefit from:}

- Convenient online submission

- Rigorous peer review

- Immediate publication on acceptance

- Open access: articles freely available online

- High visibility within the field

- Retaining the copyright to your article

Submit your next manuscript at $>$ springeropen.com 